\title{
A novel prediction model for the completion of six cycles of radium-223 treatment and survival in patients with metastatic castration-resistant prostate cancer
}

\author{
Yasuhide Miyoshi ${ }^{1} \mathbb{0}$. Sohgo Tsutsumi ${ }^{1} \cdot$ Masato Yasui $^{1} \cdot$ Takashi Kawahara $^{1} \cdot$ Ko-ichi Uemura $^{1} \cdot$ Naruhiko Hayashi $^{2}$. \\ Masahiro Nozawa ${ }^{3} \cdot$ Kazuhiro Yoshimura $^{3} \cdot$ Hiroji Uemura $\cdot$ Hirotsugu Uemura ${ }^{3}$
}

Received: 17 December 2020 / Accepted: 14 February 2021 / Published online: 1 March 2021

(c) The Author(s) 2021

\begin{abstract}
Purpose We evaluated the predictive factors for completion of all six cycles of radium-223 (Ra-223) treatment in patients with metastatic castration-resistant prostate cancer (mCRPC). We also developed a novel prediction model for Ra-223 treatment completion using these predictors.

Methods We retrospectively reviewed data from 122 patients with mCRPC who were treated with Ra-223. The predictive factors for the completion of six cycles of Ra-223 treatment were evaluated. Statistically significant predictive factors were then used to develop a prediction model for treatment completion. Finally, using this prediction model, we classified the overall survival (OS) of the entire cohort into three groups.

Results We identified three significant variables as the predictive factors for treatment completion: baseline alkaline phosphatase (ALP) level, baseline hemoglobin $(\mathrm{Hb})$ level, and baseline pain. The three groups generated using the prediction model were: group 1 (patients with three predictive factors, i.e., ALP $<$ median, $\mathrm{Hb} \geq$ median, and no pain), group 2 (patients with one to two predictive factors), and group 3 (patients without any predictive factors). The treatment completion rates differed between the three groups significantly. Furthermore, the OS also differed among the groups significantly.

Conclusion Our study suggested that the baseline ALP level, baseline $\mathrm{Hb}$ level, and baseline pain were the predictive factors of completion of all six cycles of Ra-223 treatment in patients with mCRPC. Our prediction model consisting of these factors could predict not only the completion of Ra-223 treatment, but also the post-treatment survival. This model can thus be useful for selection of patients for Ra-223 treatment.
\end{abstract}

Keywords Prostate cancer $\cdot$ Radium-223 $\cdot$ Hemoglobin $\cdot$ Alkaline phosphatase $\cdot$ Pain

Yasuhide Miyoshi

miyoyasu@med.yokohama-cu.ac.jp

1 Department of Urology and Renal Transplantation, Yokohama City University Medical Center, 4-57 Ufafune-cho Minami-ku, Yokohama, Kanagawa 232-0024, Japan

2 Department of Urology, Yokohama City University Graduate School of Medicine, 3-9 Fukuura Kanazawa-ku, Yokohama, Kanagawa 236-0004, Japan

3 Department of Urology, Kindai University Faculty of Medicine, 377-2 Ohno-higashi, Osakasayama, Osaka 589-8511, Japan

\section{Introduction}

Radium-223 (Ra-223) is an alpha-emitting radionuclide that selectively targets bone metastases [1]. The Alpharadin in Symptomatic Prostate Cancer Patients (ALSYMPCA) study (a phase III, randomized, double-blind, and multinational study) demonstrated that Ra-223 treatment led to an improvement in not only the survival, but also the healthrelated quality of life of patients with metastatic castrationresistant prostate cancer (mCRPC) [1-3]. In this study, the median overall survival (OS) of patients treated with Ra-223 and the placebo was 14.0 and 11.2 months, respectively, and the hazard ratio (HR) was 0.70 (95\% confidence interval [CI] 0.55-0.88).

Based on these findings, Ra-223 was established as a standard treatment modality for mCRPC patients with 
predominant bone disease and no visceral metastases [4-6]. The approved regimen of Ra-223 is an intravenous injection of $55 \mathrm{kBq} / \mathrm{kg}$ of Ra-223, every 4 weeks (q4w), for six cycles $[1,4]$.

The biological effectiveness of a radionuclide therapy is dose-dependent [7]. Saad et al. conducted a phase IIIb, single-arm study in the setting of an international early access program (iEAP) [8] that evaluated the correlation between the number of treatment cycles of Ra-223 (1-4 vs. 5-6) and the OS using a post hoc analysis [7]. The primary endpoints of this study were the safety and survival of patients treated with Ra-223 for symptomatic or asymptomatic mCRPC. It was found that patients who completed the treatment achieved a better clinical outcome (5-6 cycles; median OS: not reached) than those who discontinued early (1-4 cycles; median OS: 6.3 months). Therefore, completion of Ra-223 treatment is necessary for improving survival. However, the predictors for Ra-223 treatment completion have not been established yet. Therefore, in this study, we evaluated the predictors for completion of six cycles of Ra-223 treatment in $\mathrm{mCRPC}$ patients and also developed a novel prediction model for the same.

\section{Patients and methods}

\section{Patients}

We retrospectively reviewed 122 patients with bone-mCRPC who were treated with Ra-223 between 2012 and 2020 at the Yokohama City University Medical Center and the Kindai University Hospital. Some patients had participated in the Japanese trial on Ra-223. All patients presented with histologically confirmed conventional prostate adenocarcinoma. Patients with visceral metastasis and/or regional lymph node metastases measuring greater than $4 \mathrm{~cm}$ were excluded. Patients were administered with Ra-223 intravenously at a dose of $55 \mathrm{kBq} / \mathrm{kg}, \mathrm{q} 4 \mathrm{w}$, for up to six cycles. Additionally, all patients were also administered with a luteinizing hormone-releasing hormone (LHRH) agonist or antagonist. In some patients, abiraterone acetate (ABI) was administered simultaneously with Ra-223 before the results of the phase III ERA-223 trial were obtained; this trial demonstrated that compared to ABI alone, the combination of Ra-223 and $\mathrm{ABI}$ increased the incidence of bone fracture [9]. Furthermore, bone-modifying agents (BMAs) such as zoledronic acid (4 mg) or denosumab (120 mg) were also administered (qw4) in accordance with the physicians' judgement.

Prostate-specific antigen (PSA) levels and laboratory data were evaluated $\mathrm{q} 4 \mathrm{w}$, and computed tomography and/or bone scanning with $99 \mathrm{~m}$-technetium were performed every 12 weeks. In cases of cancer progression or severe adverse events, the physicians ceased the Ra-223 treatment before six cycles were completed and changed it.

After Ra-223 failure, all patients continued to receive LHRH agonist or antagonist, and were subsequently treated with ABI, enzalutamide (ENZ), docetaxel, cabazitaxel, steroids, and/or the best supportive care, in accordance with each physician's treatment strategy.

All experimental procedures were conducted in accordance with the ethical standards of the Helsinki Declaration. This study was approved by the institutional review board of the Yokohama City University Medical Center (B181000040). Informed consent for participation in this study was obtained from all subjects in an opt-out manner.

\section{Clinical assessments}

The following data were collected from electronic medical records: the patient's age; concurrent use of ABI, ENZ, and BMA; baseline alkaline phosphatase (ALP), hemoglobin $(\mathrm{Hb})$, lactate dehydrogenase (LDH), and PSA levels; baseline pain; history of docetaxel use; number of Ra-223 treatment cycles; and survival. Patients without pain were included in this study, because Ra-223 was approved for both, mCRPC patients with and without pain in Japan. CRPC was defined according to the Prostate Cancer Working Group 2 criteria [10]. Pain was defined by the patient's subjective judgement.

\section{Statistical analysis}

All analyses were conducted using IBM SPSS Statistics software for Windows, version 26 (IBM Corp., Armonk, NY, USA) and EZR (Saitama Medical Center, Jichi Medical University, Saitama, Japan) [11]. Data on continuous variables were divided into binary variables based on the median value of each variable.

The Fisher's exact test was to evaluate the distribution of the frequency of each variable in different groups, namely, patients with treatment completion and patients without treatment completion). The Mann-Whitney $U$ test was used to compare the differences in the running variables between those two groups.

The primary endpoints of this study were the predictive factors of completion of six cycles of Ra-223 treatment. Multivariate analysis using a logistic regression model was used to extract significant predictive factors for completion of six cycles of Ra-223 treatment, and the OR and 95\% CI for each variable were determined. On the basis of statistically significant predictive factors, we developed a prediction model for treatment completion; this model was used to classify the entire cohort into three groups.

The Kaplan-Meier product-limit estimator was used to assess the OS distribution, and the log-rank test was used 
to analyze the differences in the OS between the groups. All tests were two-sided, and $p<0.0 .5$ was considered as statistically significant.

\section{Results}

The patients' characteristics are listed in Table 1. The median observation time was 11.0 months (range 0.6-56.5 months).

Of the 122 patients analyzed, 83 patients $(68.0 \%)$ completed all six cycles of Ra-223 treatment. Of the remaining 39 patients, 8 (6.6\%), 5 (4.1\%), 5 (4.1\%), 9 (7.4\%), and $12(9.8 \%)$ patients completed one, two, three, four, and five cycles, respectively. The reasons for completing five or less cycles of treatment were cancer progression in 30 patients (PSA progression, worsening of general health condition, bone metastasis progression, increased pain, new visceral metastasis in the liver, new visceral metastasis in the lung, and cancer-related death in $6,13,6,2,1,1$, and 1 patients, respectively), medical events (except prostate cancer progression) in 3 patients (cerebrovascular events, arrhythmia, and anemia in one patient each), patient's desire in 2 patients, and unknown causes in 2 patients.

We identified three variables as the significant predictive factors for the completion of Ra-223 treatment, namely the baseline ALP level, baseline $\mathrm{Hb}$ level, and baseline pain (Table 2). Furthermore, we also developed a prediction model for treatment completion consisting of these three variables. This model could classify the entire cohort into three groups: group 1 involved patients with all three predictive factors (i.e., patients with ALP $<$ median, $\mathrm{Hb} \geq$ median, and no pain) $(n=23)$; group 2 , patients with one to two predictive factors $(n=81)$; and group 3 , patients without any predictive factors $(n=18)$.

Table 1 Patient characteristics

\begin{tabular}{|c|c|c|c|c|}
\hline & Entire cohort $(n=122)$ & $\begin{array}{l}\text { Patients with treatment } \\
\text { completion }(n=83)\end{array}$ & $\begin{array}{l}\text { Patients without treatment } \\
\text { completion }(n=39)\end{array}$ & $p$ value* \\
\hline Median age (range), years & $75(49-92)$ & $76(57-92)$ & $74(49-90)$ & 0.273 \\
\hline Concomitant use of ARTA, $n, \%$ & $40(32.8)$ & $34(41.0)$ & $6(15.4)$ & 0.007 \\
\hline Median baseline ALP level (range), IU/L & $328(113-3,540)$ & $275(113-2,584)$ & $562(163-3540)$ & $<0.001$ \\
\hline Median baseline $\mathrm{Hb}$ level (range), g/dL & $12.3(7.5-15.5)$ & $12.6(7.5-15.5)$ & $10.8(8.0-15.2)$ & $<0.001$ \\
\hline Median baseline LDH level (range), IU/L & $213(110-528)$ & $208(111-512)$ & $221(110-528)$ & 0.366 \\
\hline Median baseline PSA level (range), $\mathrm{ng} / \mathrm{mL}$ & $35.9(0.0-3157.0)$ & $23.1(0.0-859.0)$ & $79.3(2.9-3157.0)$ & $<0.001$ \\
\hline Concomitant use of BMA; $n(\%)$ & $46(37.7)$ & $32(38.6)$ & $14(35.9)$ & 0.843 \\
\hline Baseline pain; $n(\%)$ & $52(42.6)$ & $29(34.9)$ & $23(59.0)$ & 0.018 \\
\hline Previous use of docetaxel, $n(\%)$ & $42(34.4)$ & $27(32.5)$ & $15(38.5)$ & 0.545 \\
\hline
\end{tabular}

$A R T A$ androgen receptor-targeted agent, $A L P$ alkaline phosphatase, $H b$ hemoglobin, $L D H$ lactate dehydrogenase, $P S A$ prostate-specific antigen, $B M A$ bone-modifying agent

*The difference between patients with treatment completion and those without treatment completion

Table 2 Univariate and multivariate analyses for predictive factors of completion of six cycles of radium-223 treatment

\begin{tabular}{|c|c|c|c|c|c|c|c|c|}
\hline & \multicolumn{4}{|c|}{ Univariate analysis } & \multicolumn{4}{|c|}{ Multivariate analysis } \\
\hline & \multirow[t]{2}{*}{ OR } & \multicolumn{2}{|l|}{$95 \% \mathrm{CI}$} & \multirow[t]{2}{*}{$p$ value } & \multirow[t]{2}{*}{ OR } & \multicolumn{2}{|c|}{$95 \% \mathrm{CI}$} & \multirow[t]{2}{*}{$p$ value } \\
\hline & & Lower & Upper & & & Lower & Upper & \\
\hline Concomitant use of ARTA (yes vs. no) & 0.262 & 0.099 & 0.694 & 0.007 & 0.338 & 0.112 & 1.015 & 0.053 \\
\hline Median baseline ALP levels (>328 IU/L vs. $\leq 328$ IU/L) & 4.057 & 1.777 & 9.264 & 0.001 & 3.225 & 1.117 & 9.311 & 0.030 \\
\hline Median baseline Hb levels ( $>12.3 \mathrm{~g} / \mathrm{dL}$ vs. $\leq 12.3 \mathrm{~g} / \mathrm{dL}$ ) & 5.889 & 2.469 & 14.044 & $<0.001$ & 7.156 & 2.407 & 21.274 & $<0.001$ \\
\hline Median baseline LDH levels ( $>285 \mathrm{IU} / \mathrm{L}$ vs $\leq 285 \mathrm{IU} / \mathrm{L}$ ) & 1.533 & 0.712 & 3.297 & 0.275 & 1.121 & 0.398 & 3.161 & 0.829 \\
\hline Median baseline PSA levels ( $>35.9 \mathrm{ng} / \mathrm{mL}$ vs $\leq 35.9 \mathrm{ng} / \mathrm{mL}$ ) & 3.243 & 1.445 & 7.279 & 0.004 & 2.604 & 0.939 & 7.219 & 0.066 \\
\hline Concomitant use of BMA (yes vs. no) & 0.893 & 0.405 & 1.966 & 0.778 & 0.355 & 0.115 & 1.098 & 0.072 \\
\hline Baseline pain (yes vs. no) & 2.677 & 1.225 & 5.848 & 0.014 & 3.004 & 1.112 & 8.116 & 0.030 \\
\hline Previous use of docetaxel (yes vs. no) & 1.296 & 0.587 & 2.862 & 0.521 & 0.520 & 0.175 & 1.546 & 0.240 \\
\hline
\end{tabular}

$A R T A$ androgen receptor-targeted agent, $A L P$ alkaline phosphatase, $H b$ hemoglobin, $L D H$ lactate dehydrogenase, $P S A$ prostate-specific antigen, $B M A$ bone-modifying agent, $O R$ odds ratio, $C I$ confidence interval 
According to our prediction model, the completion rates of Ra-223 treatment in groups 1, 2, and 3 were 100\% (23/23), $67.9 \%$ (55/81), and $27.8 \%$ (5/18), respectively. There were statistically significant differences in the completion rates between each group (group 1 vs. group 2: $p<0.001$, group 1 vs. group 3: $p<0.001$, and group 2 vs. group 3: $p=0.002$ ).

The median survival of the entire cohort was 23.5 months (95\% CI 16.7-32.5 months) (Supplementary Fig. 1). The Kaplan-Meier curves according to treatment completion are shown in Fig. 1. The median OS differed significantly between patients who completed and did not complete the treatment and was 32.5 months and 8.8 months, respectively, $(p<0.001)$.

The Kaplan-Meier curves according to the baseline ALP level, baseline $\mathrm{Hb}$ level, and baseline pain are shown in Supplementary Figs. 2, 3, and 4, respectively. The median OS of the patients with ALP $<$ median, ALP $>$ median, $\mathrm{Hb} \geq$ median, $\mathrm{Hb}<$ median, without pain, and with pain was 32.8 months (95\% CI 22.4-not available [NA]), 15.5 months (95\% CI 10.6-23.7 months), 32.5 months (95\% CI 18.9-NA), 15.5 months (95\% CI 10.6-23.7 months), 34.8 months (95\% CI 23.5-NA), and 15.1 months (95\% CI 10.1-22.4 months), respectively.

Patients who presented with ALP $<$ median, $\mathrm{Hb} \geq$ median, and no pain had a significantly longer survival than those who did not.

Finally, the Kaplan-Meier curves according to our prediction model are shown in Fig. 2. According to this model, the median OS of the patients in groups 1,2 , and 3 was 36.0 months (95\% CI 32.5-NA), 22.4 months (95\% CI
15.1-32.8 months), and 10.8 months (95\% CI 5.5-NA), respectively. There were significant differences in the OS between all groups (group 1 vs. group 2: $p=0.021$, group 1 vs. group 3: $p<0.001$, and group 2 vs. group 3: $p=0.018$ ).

\section{Discussions}

To improve the oncological outcomes after Ra-223 treatment, the completion of all six cycles of treatment is required $[7,12]$. Therefore, it is necessary to identify the predictive factors for the completion of Ra-223 treatment. Saad et al. reported that patients treated with 5-6 cycles of Ra-223 treatment had a longer OS than those treated with 1-4 cycles (median OS: not reached vs. 6.3 months). Furthermore, less pain (non-mild), a good Eastern Cooperative Oncology Group performance status (0-1), lower PSA level $(\leq 141 \mathrm{ng} / \mathrm{mL})$, and higher $\mathrm{Hb}(\geq 10 \mathrm{~g} / \mathrm{dL})$ were the predictive factors for completing five to six cycles of treatment [7]. In a population-based study, Parimi et al. also reported that patients in the $\geq$ five cycles group had a longer OS as compared to those in the < five cycles group (median OS 16.2 vs. 5.9 months). They reported that a lower ALP level $(<220 /$ $\mathrm{IU} / \mathrm{L})$ and higher $\mathrm{Hb}$ level $(>11.8 \mathrm{~g} / \mathrm{dL})$ were the predictive factors for completing from five to six cycles of treatment [12]. Our retrospective study also demonstrated that patients treated with six cycles of treatment had a significantly longer OS as compared to those treated with five or fewer cycles of treatment. Moreover, multivariate analysis demonstrated that ALP level $<$ median, $\mathrm{Hb}$ level $\geq$ median, and no pain
Fig. 1 The Kaplan-Meier curves for overall survival according to Ra-223 treatment completion. The black and red lines indicate the survival of patients with and without completion, respectively

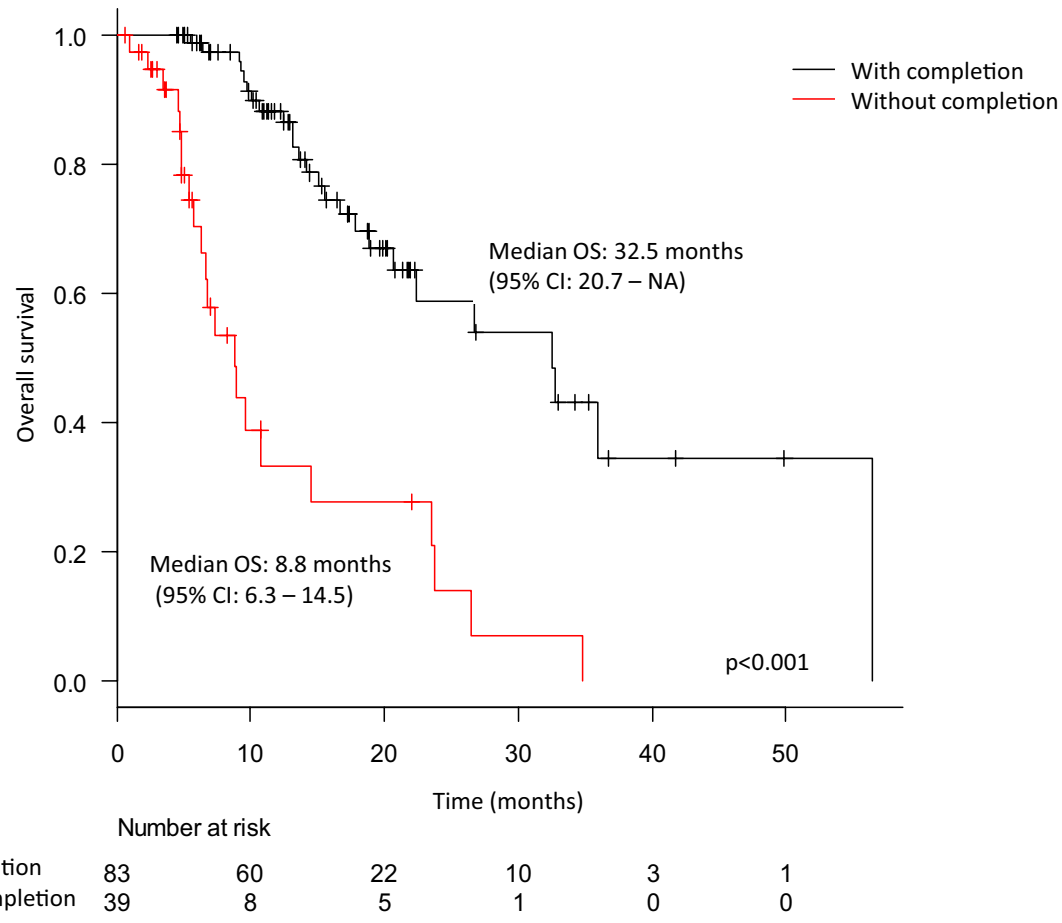


Fig. 2 The Kaplan-Meier curves for overall survival according to our predictive model consisting of the baseline alkaline phosphatase (ALP) levels, hemoglobin ( $\mathrm{Hb})$ levels, and pain. The black, red, and green lines indicate the survival of patients in group 1 , patients with all three predictive factors (i.e., patients with $\mathrm{ALP}<$ median, $\mathrm{Hb} \geq$ median, and no pain), group 2 , patients with one to two predictive factors, and group 3, patients without any predictive factors, respectively

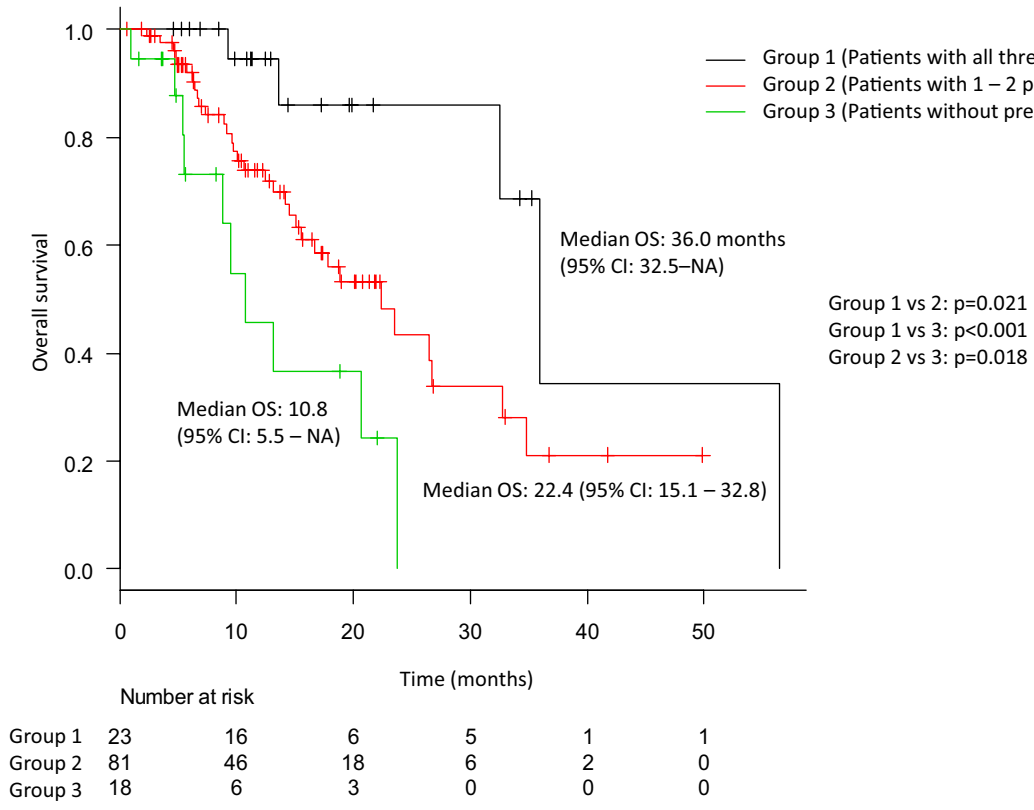

Group 1 vs 2: $p=0.021$

Group 318

\section{,}

were the significant predictive factors for Ra-223 treatment completion. Based on these results, we developed a predictive model for the completion of Ra-223 treatment consisting of ALP, $\mathrm{Hb}$, and pain. Our model could stratify the entire cohort into three groups according to the number of predictive factors. It could predict not only the completion of Ra-223 treatment, but also the survival after Ra-223 treatment.

This study had some limitations. Ra-223 is only approved for symptomatic CRPC patients by the US Food and Drug administration [13] and European Medicines Agency [14]. Therefore, the applicability of our model in patients with asymptomatic CRPC in the US and European countries remains unclear. However, despite the retrospective nature of our study and the small number of patients, our findings indicate that the $\mathrm{Hb}$ and ALP levels should be considered when the physician decides upon the use of Ra-223 in patients with mCRPC. For the confirmation of our results, a prospective study is warranted in the future.

\section{Conclusions}

Our retrospective study suggested that the baseline ALP level, baseline $\mathrm{Hb}$ level, and baseline pain were the predictive factors for completion of six cycles of Ra-223 treatment in patients with mCRPC. Our prediction model consisting of these three predictors could predict not only the completion of six cycles of Ra-223, but also the survival post-treatment. Therefore, our prediction model could be useful for selecting patients for Ra-223 treatment.
Supplementary Information The online version contains supplementary material available at https://doi.org/10.1007/s00345-021-03639-z.

Acknowledgements We would like to thank Dr. Yumiko Yokomizo at the Department of Urology, Yokohama City University Hospital, Dr. Naoki Matsumura, Dr. Takayuki Ohzeki, and Dr. Takafumi Minami from the Department of Urology, Kindai University Hospital for collecting patients' data.

Author contributions YM: protocol/project development, data collection or management, data analysis, manuscript writing. ST: data collection or management. MY: data collection or management. TK: data collection or management. KU: data collection or management. $\mathrm{NH}$ : data collection or management. MN: data collection or management. KY: data collection or management. HU: protocol/project development, data collection or management. HU: protocol/project development, data collection or management.

Funding None.

Availability of data and materials The datasets generated during and/or analysed during the current study are available from the corresponding author on reasonable request.

\section{Compliance with ethical standards}

Conflict of interest The authors have nothing to disclose.

Open Access This article is licensed under a Creative Commons Attribution 4.0 International License, which permits use, sharing, adaptation, distribution and reproduction in any medium or format, as long as you give appropriate credit to the original author(s) and the source, provide a link to the Creative Commons licence, and indicate if changes were made. The images or other third party material in this article are included in the article's Creative Commons licence, unless indicated otherwise in a credit line to the material. If material is not included in the article's Creative Commons licence and your intended use is not permitted by statutory regulation or exceeds the permitted use, you will 
need to obtain permission directly from the copyright holder. To view a copy of this licence, visit http://creativecommons.org/licenses/by/4.0/.

\section{References}

1. Parker C, Nilsson S, Heinrich D, Helle SI, O'Sullivan JM, Fosså SD, Chodacki A, Wiechno P, Logue J, Seke M, Widmark A, Johannessen DC, Hoskin P, Bottomley D, James ND, Solberg A, Syndikus I, Kliment J, Wedel S, Boehmer S, Dall'Oglio M, Franzén L, Coleman R, Vogelzang NJ, O’Bryan-Tear CG, Staudacher K, Garcia-Vargas J, Shan M, Bruland ØS, Sartor O (2013) Alpha emitter radium-223 and survival in metastatic prostate cancer. N Engl J Med 369(3):213-223. https://doi.org/10.1056/NEJMo a1213755

2. Sartor O, Coleman R, Nilsson S, Heinrich D, Helle SI, O'Sullivan JM, Fossa SD, Chodacki A, Wiechno P, Logue J, Widmark A, Johannessen DC, Hoskin P, James ND, Solberg A, Syndikus I, Vogelzang NJ, O'Bryan-Tear CG, Shan M, Bruland OS, Parker C (2014) Effect of radium-223 dichloride on symptomatic skeletal events in patients with castration-resistant prostate cancer and bone metastases: results from a phase 3, double-blind, randomised trial. Lancet Oncol 15(7):738-746. https://doi.org/10.1016/s1470 -2045(14)70183-4

3. Nilsson S, Cislo P, Sartor O, Vogelzang NJ, Coleman RE, O'Sullivan JM, Reuning-Scherer J, Shan M, Zhan L, Parker C (2016) Patient-reported quality-of-life analysis of radium-223 dichloride from the phase III ALSYMPCA study. Ann Oncol 27(5):868-874. https://doi.org/10.1093/annonc/mdw065

4. Takahashi S, Kakehi Y, Masumori N, Hosono M, Kinuya S, Okayama Y, Sunaya T, Okumura M, Uemura H (2020) Safety and effectiveness of radium-223 dichloride (Ra-223) in patients with mCRPC in real-world setting: a Japanese post-marketing study (PMS). J Clin Oncol 38(6):236

5. Shore N, Higano CS, George DJ, Sternberg CN, Saad F, Tombal B, Miller K, Kalinovsky J, Jiao X, Tangirala K, Sartor O (2020) Concurrent or layered treatment with radium-223 and enzalutamide or abiraterone/prednisone: real-world clinical outcomes in patients with metastatic castration-resistant prostate cancer. Prostate Cancer Prostatic Dis. https://doi.org/10.1038/s4139 1-020-0236-0

6. Hashimoto K, Miyoshi Y, Shindo T, Hori M, Tsuboi Y, Kobayashi K, Fukuta F, Tanaka T, Miyamoto S, Maehana T, Okada M, Nishiyama N, Yanase M, Kato R, Hotta H, Kunishima Y, Takahashi A, Hinotsu S, Sakata KI, Kitamura H, Uemura H, Masumori N (2020) Dynamic changes of bone metastasis predict bone-predominant status to benefit from radium-223 dichloride for patients with castration-resistant prostate cancer. Cancer Med. https://doi. org/10.1002/cam4.3459

7. Saad F, Gillessen S, Heinrich D, Keizman D, O'Sullivan JM, Nilsson S, Miller K, Wirth M, Reeves J, Seger M, Carles J, Heidenreich A (2019) Disease characteristics and completion of treatment in patients with metastatic castration-resistant prostate cancer treated with radium-223 in an international early access program. Clin Genitourin Cancer 17(5):348.e345-355.e345. https ://doi.org/10.1016/j.clgc.2019.05.012

8. Saad F, Carles J, Gillessen S, Heidenreich A, Heinrich D, Gratt J, Levy J, Miller K, Nilsson S, Petrenciuc O, Tucci M, Wirth M, Federhofer J, O'Sullivan JM, Radium-223 International Early Access Program I (2016) Radium-223 and concomitant therapies in patients with metastatic castration-resistant prostate cancer: an international, early access, open-label, single-arm phase $3 \mathrm{~b}$ trial. Lancet Oncol 17(9):1306-1316. https://doi.org/10.1016/s1470 -2045(16)30173-5

9. Smith M, Parker C, Saad F, Miller K, Tombal B, Ng QS, Boegemann M, Matveev V, Piulats JM, Zucca LE, Karyakin O, Kimura G, Matsubara N, Nahas WC, Nolè F, Rosenbaum E, Heidenreich A, Kakehi Y, Zhang A, Krissel H, Teufel M, Shen J, Wagner V, Higano C (2019) Addition of radium-223 to abiraterone acetate and prednisone or prednisolone in patients with castration-resistant prostate cancer and bone metastases (ERA 223): a randomised, double-blind, placebo-controlled, phase 3 trial. Lancet Oncol 20(3):408-419. https://doi.org/10.1016/s1470-2045(18)30860-x

10. Scher HI, Halabi S, Tannock I, Morris M, Sternberg CN, Carducci MA, Eisenberger MA, Higano C, Bubley GJ, Dreicer R, Petrylak D, Kantoff P, Basch E, Kelly WK, Figg WD, Small EJ, Beer TM, Wilding G, Martin A, Hussain M (2008) Design and end points of clinical trials for patients with progressive prostate cancer and castrate levels of testosterone: recommendations of the Prostate Cancer Clinical Trials Working Group. J Clin Oncol 26(7):1148-1159. https://doi.org/10.1200/jco.2007.12.4487

11. Kanda Y (2013) Investigation of the freely available easy-to-use software "EZR" for medical statistics. Bone Marrow Transplant 48(3):452-458. https://doi.org/10.1038/bmt.2012.244

12. Parimi S, Tsang E, Alexander A, McKenzie M, Bachand F, Sunderland K, Chi KN, Aparicio M, Worsley D, Tyldesley S (2017) A population-based study of the use of radium 223 in metastatic castration-resistant prostate cancer: Factors associated with treatment completion. Can Urol Assoc J 11(10):350-355. https://doi. org/10.5489/cuaj.4415

13. Kluetz PG, Pierce W, Maher VE, Zhang H, Tang S, Song P, Liu Q, Haber MT, Leutzinger EE, Al-Hakim A, Chen W, Palmby T, Alebachew E, Sridhara R, Ibrahim A, Justice R, Pazdur R (2014) Radium Ra 223 dichloride injection: U.S. Food and Drug Administration drug approval summary. Clin Cancer Res 20(1):9-14. https://doi.org/10.1158/1078-0432.Ccr-13-2665

14. Poeppel TD, Handkiewicz-Junak D, Andreeff M, Becherer A, Bockisch A, Fricke E, Geworski L, Heinzel A, Krause BJ, Krause T, Mitterhauser M, Sonnenschein W, Bodei L, Delgado-Bolton RC, Gabriel M (2018) EANM guideline for radionuclide therapy with radium-223 of metastatic castration-resistant prostate cancer. Eur J Nucl Med Mol Imaging 45(5):824-845. https://doi. org/10.1007/s00259-017-3900-4

Publisher's Note Springer Nature remains neutral with regard to jurisdictional claims in published maps and institutional affiliations. 